\title{
Direct-Ink-Write 3D Printing of Hydrogels into Biomimetic Soft Robots
}

Yin Cheng, Kwok Hoe Chan, Xiaoqiao Wang, Tianpeng Ding, Tongtao Li, Xin

Lu, Ghim Wei Ho*

Department of Electrical and Computer Engineering, National University of

Singapore, 4 Engineering Drive 3, Singapore 117583, Singapore

Corresponding author: G. W. Ho (email: elehgw@nus.edu.sg) 


\section{Section S1. The modeling and fitting of the coiling mechanism}

To analyze the bending curvature of the PDMS-PNIPAM structure. We simplify the structure as two rectangular layer (PNIPAM and PDMS) bonded at interface. The thickness of PNIPAM and PDMS is $t_{1}$ and $t_{2}$, respectively. The elastic modulus was measured to be $E_{1}(\sim 85 \mathrm{KPa})$ and $E_{2}$ $(\sim 1.6 \mathrm{MPa})$ for PNIPAM and PDMS. The Euler-Bernoulli theory was adopted here, similar to the modeling of a heated bimetallic strip by Timoshenko. ${ }^{1}$ The Euler-Bernoulli theory holds the assumption that the plane cross-section remains plane after bending, and still perpendicular to the curved axis after bending. The bending curvature $k$ caused by the interface strain mismatch $\varepsilon$ due to the PNIPAM contraction (linear contraction ration of 0.23 ) is given as:

$k=\frac{6 \varepsilon(1+m)^{2}}{\left(t_{1}+t_{2}\right) \cdot\left[3(1+m)^{2}+(1+m n)\left(m^{2}+\frac{1}{m n}\right)\right]}$

Here, $m=t_{1} / t_{2}$, and $n=E_{1} / E_{2}$. The dependence of bending curvature on the PDMS thickness $t_{2}$ can be obtained using this expression and shown in Fig. 5b, which agrees well with the experimental results. 
a

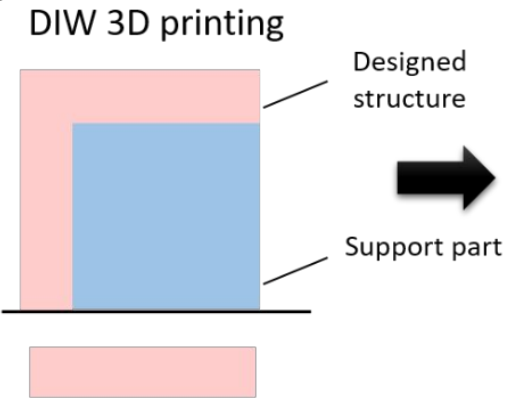

Functional DIW ink (printable and curable) b
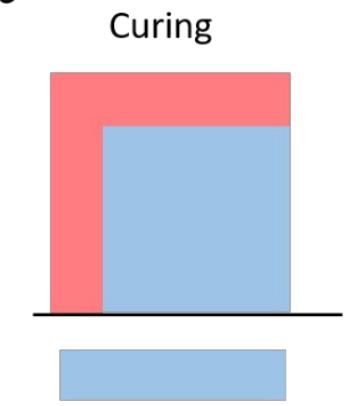

Support material

(printable and not curable)
C Support removing

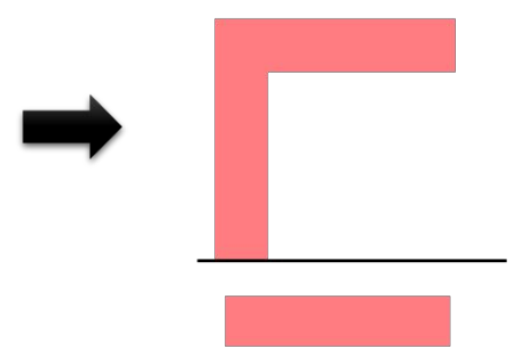

Desired design after curing

Figure S1. The schematic diagram of support printing for suspended structure in DIW 3D printing. (a) The desired suspended structure of the functional DIW ink was printed upon the support part from printable pure alginate ink (sodium alginate of $6 \mathrm{wt} \%$ and calcium chloride of $0.4 \mathrm{wt} \%$ dissolved in distilled water). (b) Curing of the functional DIW ink. (c) After curing, the support ink can be easily removed and recycled for next use, or washed away using DI water. 


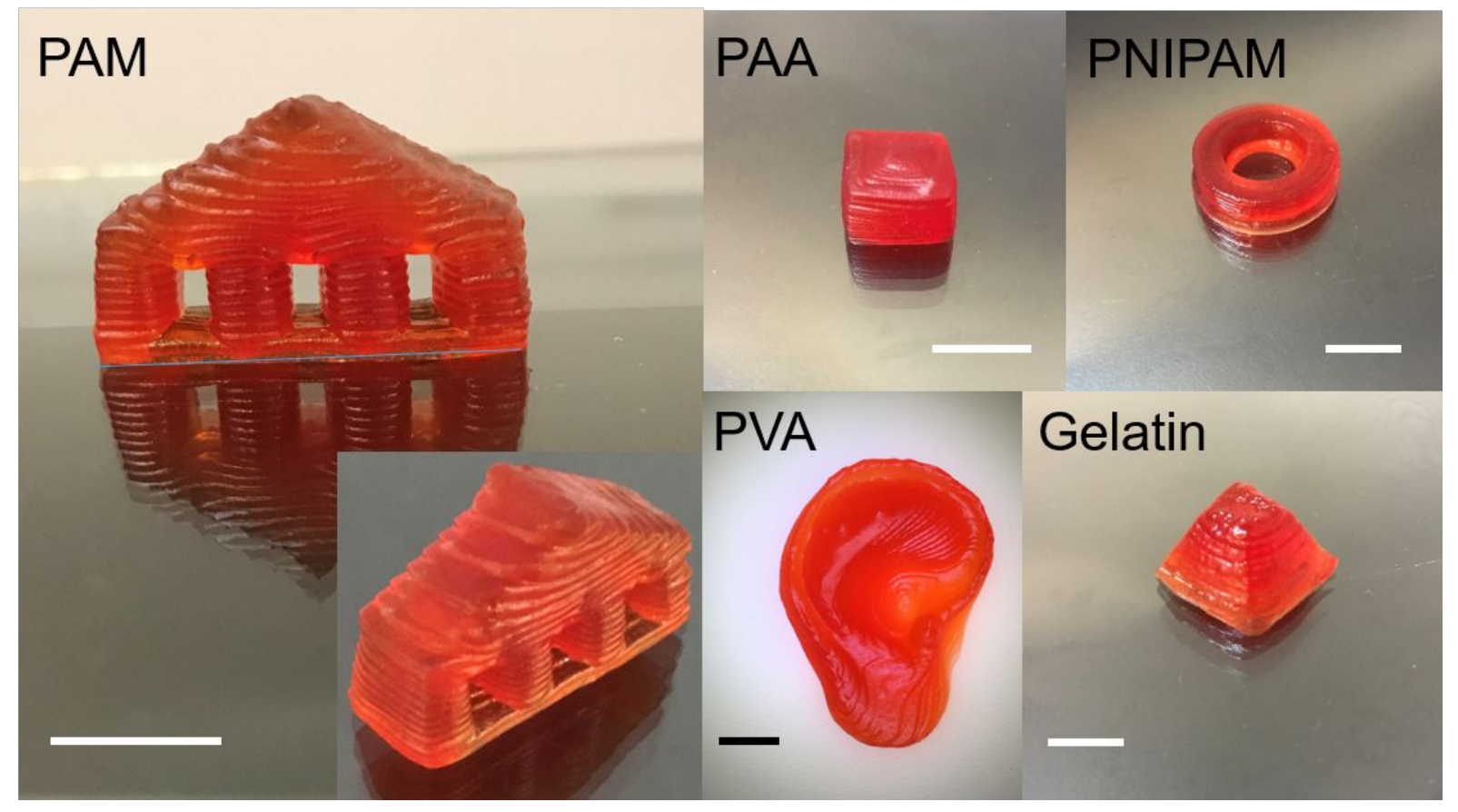

Figure S2. The printed chemically and physically crosslinked hydrogel samples. The samples were printed and cured from AAM based DIW ink (house shape), AA based DIW ink (cuboid shape), NIPAM based DIW ink (donut shape), PVA based DIW ink (ear shape), and gelatin based DIW ink (tapered pyramid shape). All the scale bars are $10 \mathrm{~mm}$. 


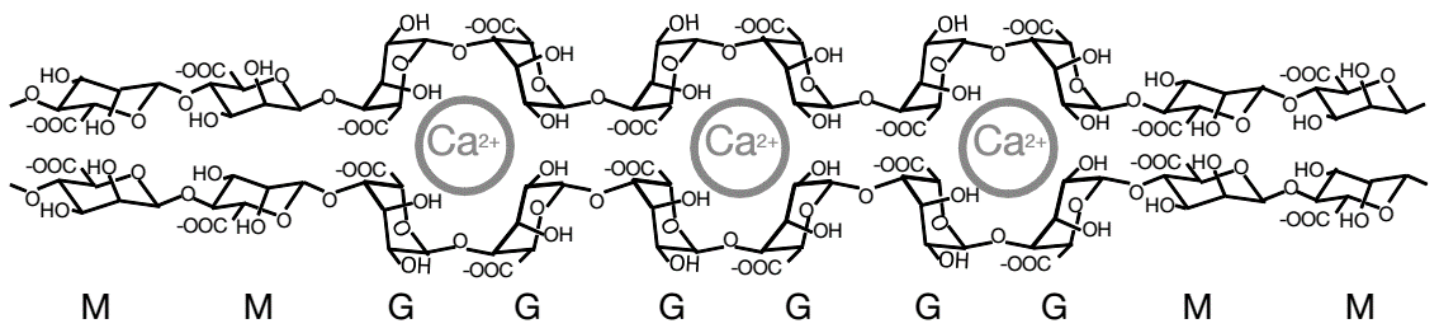

Figure S3. Schematic of the ionic crosslink between the alginate chains and calcium ions. Alginate is a linear unbranched copolymer composed of mannuronic acid (M unit) and guluronic acid ( $G$ unit), arranged in blocks rich in $G$ units, blocks rich in $M$ units, and blocks of alternating $G$ and $M$ units. The divalent cations $\left(\mathrm{Ca}^{2+}\right.$ in our work) can bind ionically to $G$ blocks, as the structure of the $G$ blocks allows high degree of coordination of the divalent ions. The $G$ blocks of one polymer then form junctions with the ones of adjacent polymer chains, to form an "egg box" model of cross-linking, resulting in a gel structure. Such ionic crosslink is mechanically reversible to exhibit rapid responsive association/dissociation, which endows the ink with a strong shear-thinning effect during the printing. 


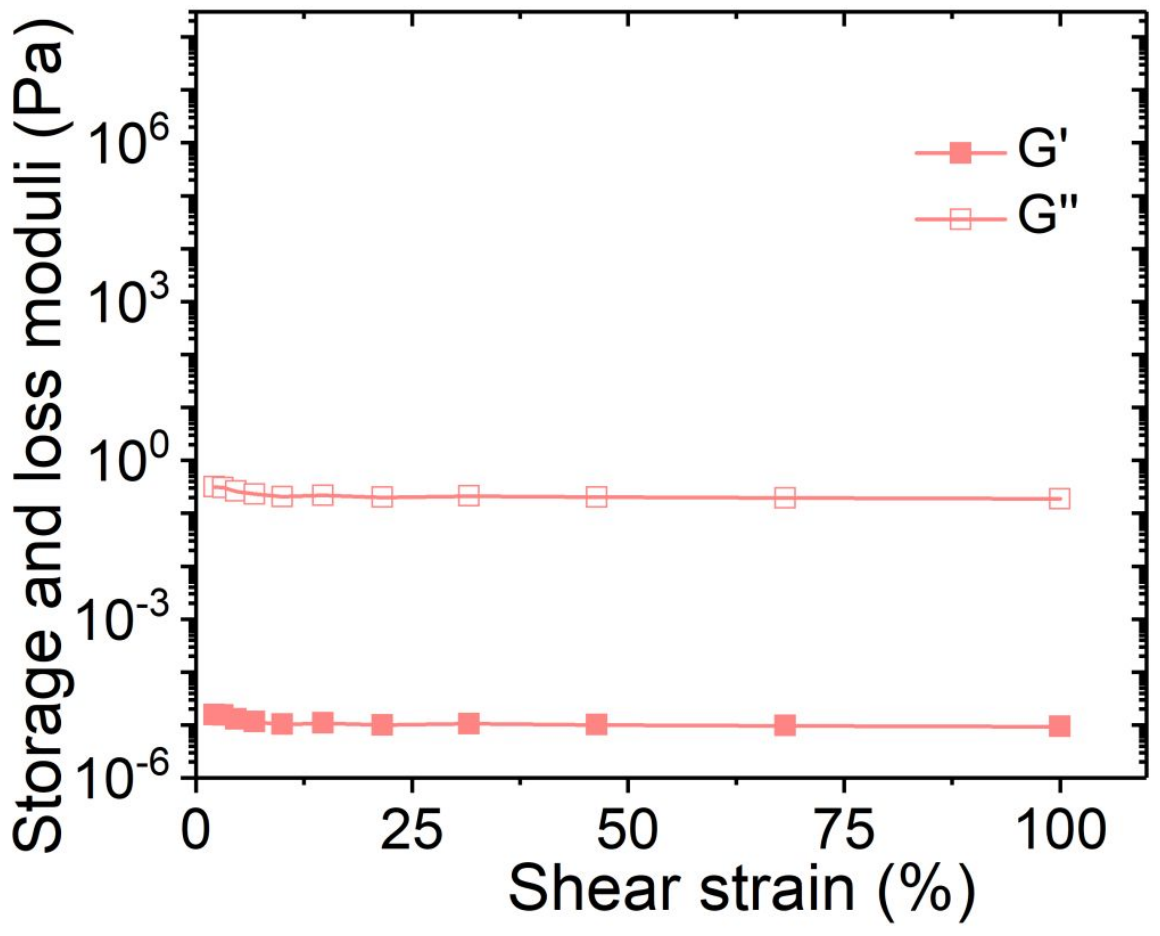

Figure S4. The oscillatory amplitude sweep test to the ink 1 at $1 \mathrm{~Hz}$. Ink 1 (no alginate addition) shows constant and much lower values of $G^{\prime}\left(\sim 1 \times 10^{-5} \mathrm{~Pa}\right)$ and $G^{\prime \prime}(\sim 0.2 \mathrm{~Pa})$ than ink $2-4$. 

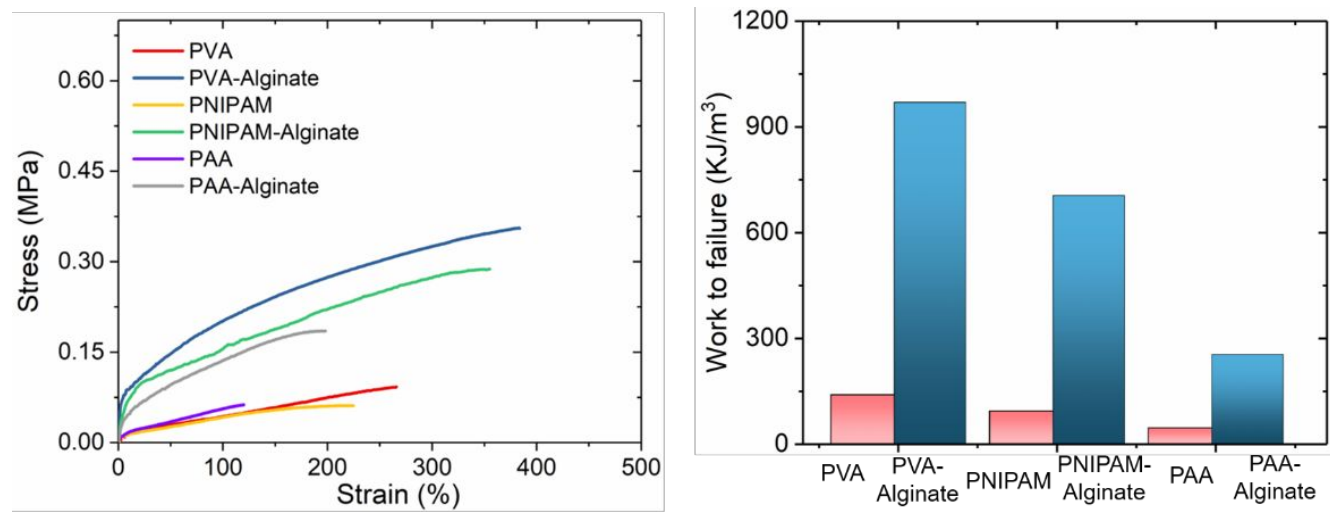

Figure S5. The mechanical performance of covalently cross-linked poly(Nisopropylacrylamide) (PNIPAM) and poly(acrylic acid) (PAA) based hydrogels, and physically crosslinked poly(vinyl alcohol) (PVA) based hydrogel, before and after adding alginate. The recipes of these hydrogels with no alginate addition are the same as the prepared inks for printing (detailed information in the Methods part), except that no alginate is used as rheological modifier. The pre-solution of the hydrogels are injected into a hollow polytetrafluoroethylene (PTFE) tube with inner diameter of $1.6 \mathrm{~mm}$ and cured. After curing, the samples are extruded out of the tube using a syringe. (a) Stress-strain curves of hydrogels (PVA, PNIPAM, PAA) before and after adding alginate. (b) The work-to-failure of hydrogels before and after adding alginate. 


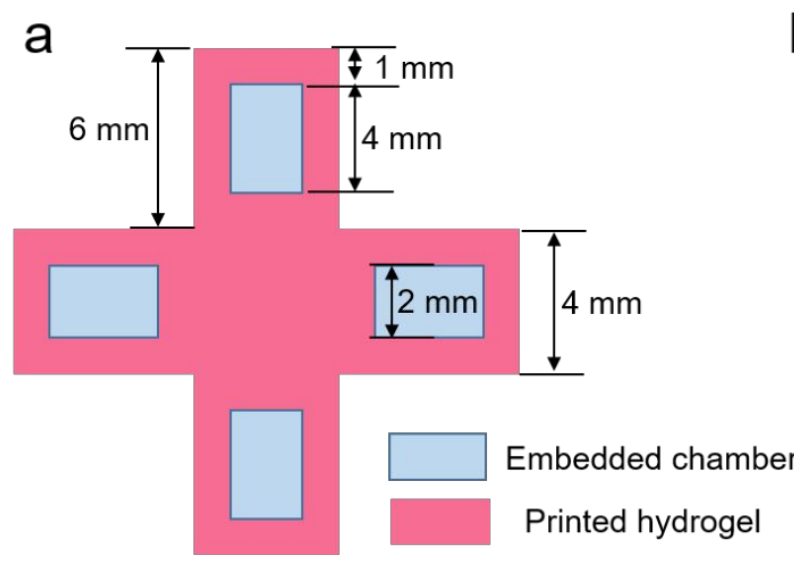

b
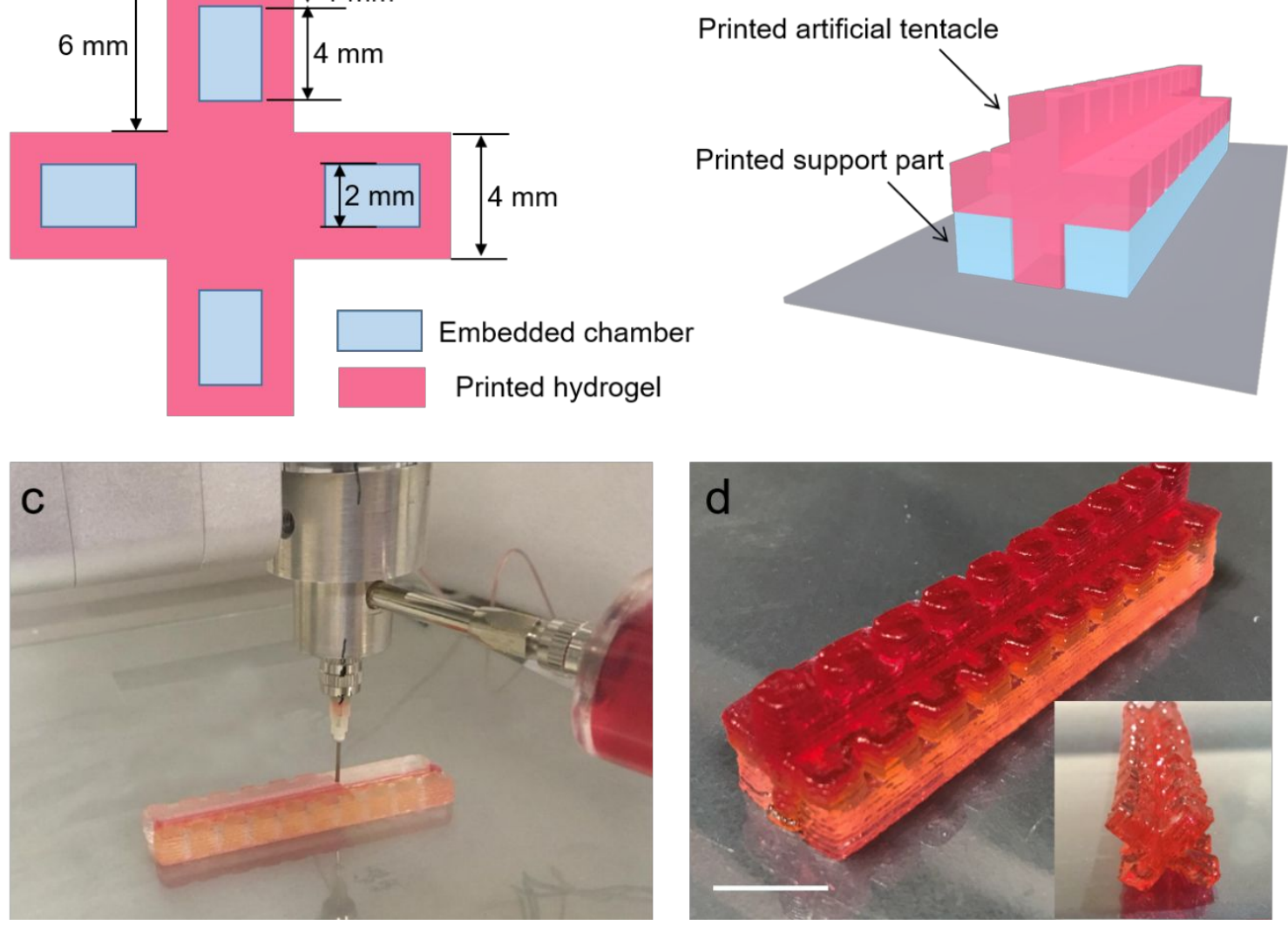

Figure S6. The geometrical details of the artificial tentacle design and the support printing aided DIW 3D printing. (a) The detailed geometry information of the artificial tentacle. (b) The schematic diagram of the support printing aided tentacle printing. (c) Photo of the tentacle printing, with transparent support part and red color AAM based ink. (d) The printed artificial tentacle with the support part after printing. Inset photo shows the printed artificial tentacle after curing and support part removing. Scale bar is $\mathbf{2} \mathrm{cm}$. During printing, the hollow channels are bridging structures with bridging distance of 2 and $4 \mathrm{~mm}$. Such bridging structures with relatively low bridging distances can be directly printed without the support material. 
a

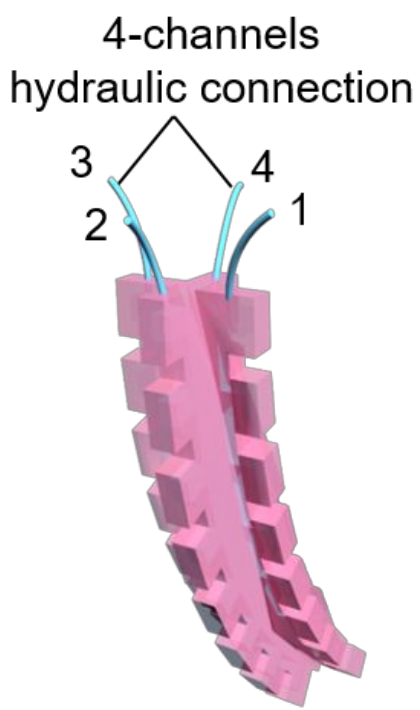

b
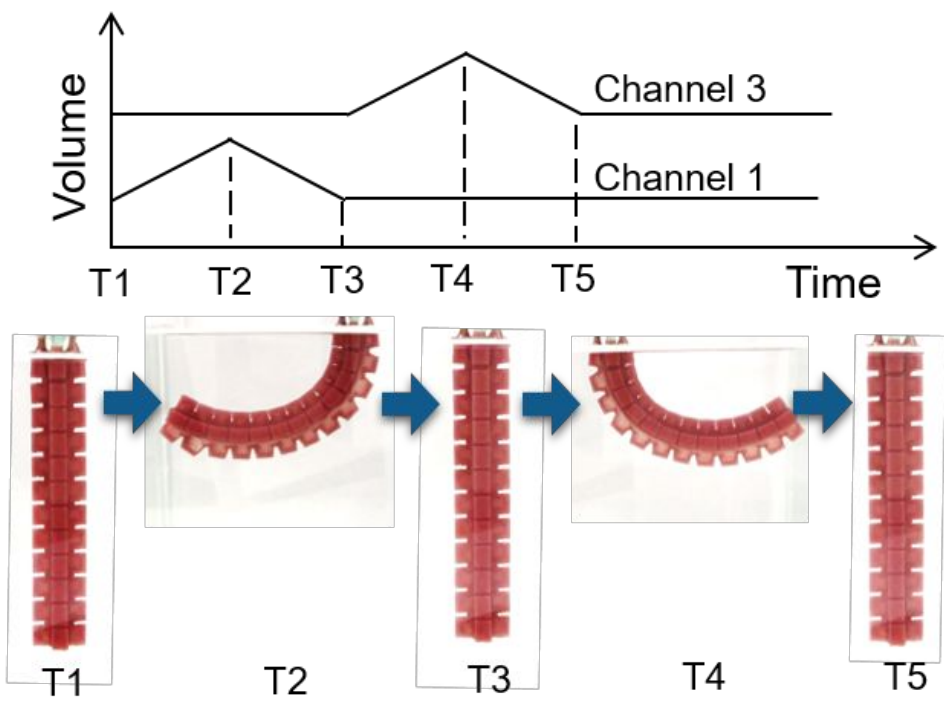

Figure S7. In-plane bending of the artificial tentacle. (a) The schematic diagram of the artificial tentacle with 4 -channels hydraulic connection. Two channels in diagonal directions are employed here. (b) The programmed time-dependent water injection into channels 1 and 3 (top) and the corresponding in-plane bending process of the artificial tentacle from time $T_{1}$ to $T_{5}$ (bottom). The peak injected volume was $1 \mathrm{ml}$ and the injection cycle time (from $T_{1}$ to $T_{3}$ ) was $4 s$. 

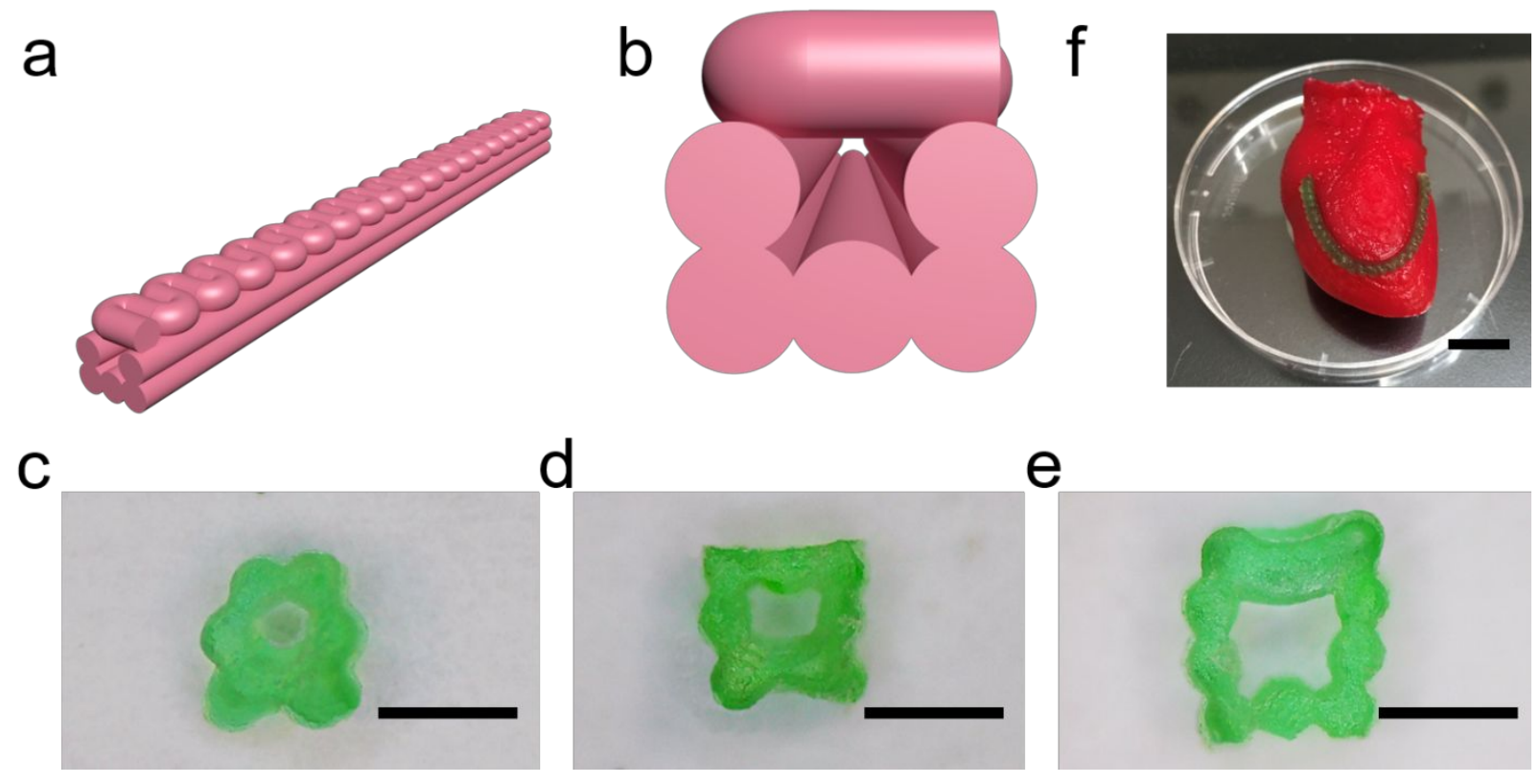

Figure S8. Printed hydrogel vessel form PVA based DIW ink. (a) The full view of the schematic hydrogel vessel from DIW 3D printing. (b) The side view of the schematic hydrogel vessel. The cross-section of micro-channel is approximately square-shaped, with tunable side length being (c) 0.3 , (d) 0.5 , and (e) $0.7 \mathrm{~mm}$. (f) Photo of the robotic heart with a hydrogel vessel (same micro-channel size as in c printed on the outer surface. The printing nozzle size is $0.51 \mathrm{~mm}$ in diameter. Here the nozzle of $0.51 \mathrm{~mm}$ in diameter was used to print filaments and construct the hollow channels. We varied the distance between the two side-wall filaments and the number of side-wall filament layer to control the width and the height of the channel's cross-section, respectively. When the side length was smaller than $0.3 \mathrm{~mm}$, the adjacent ink filaments were prone to adhere to each other during the printing. Scale bars in (c), (d), and (e) are $1 \mathrm{~mm}$. Scale bar in (f) is $1 \mathrm{~cm}$. 


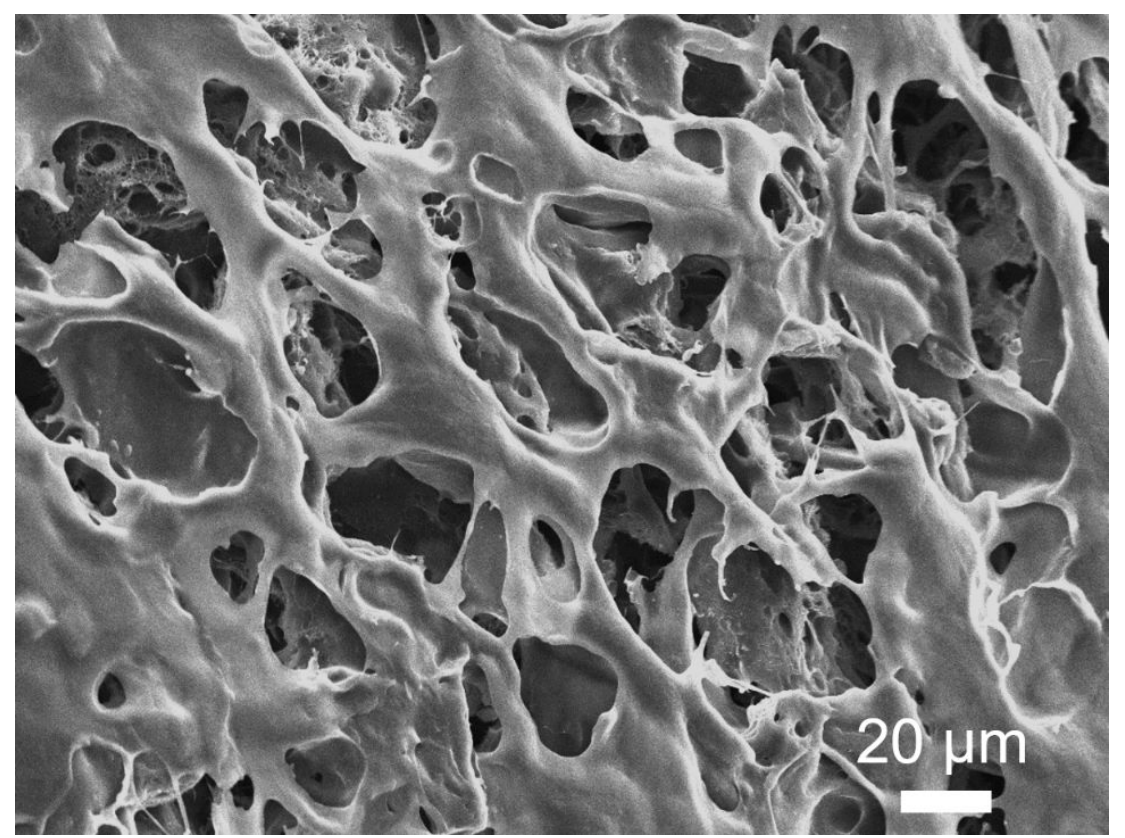

Figure S9. SEM image of the hydrogel printed from the PVA based DIW ink. A strip of hydrogel was printed from PVA based DIW ink and then cured (freeze-thaw cycles as in Materials and Methods). After curing, the sample was freeze-dried for the SEM characterization. The hydrogel morphology shows a porous structure with pore size of several tens of micrometers. 
a

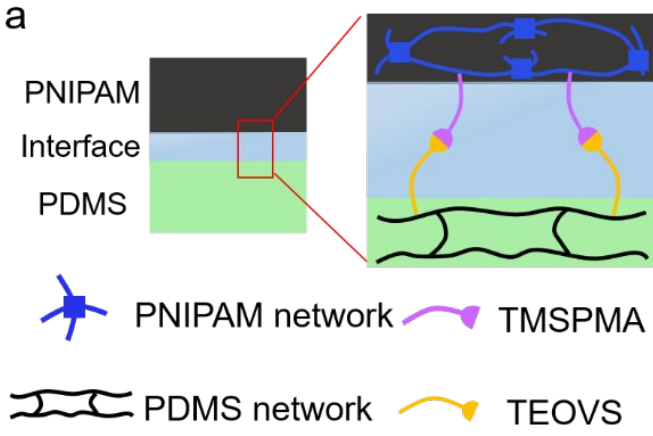

d

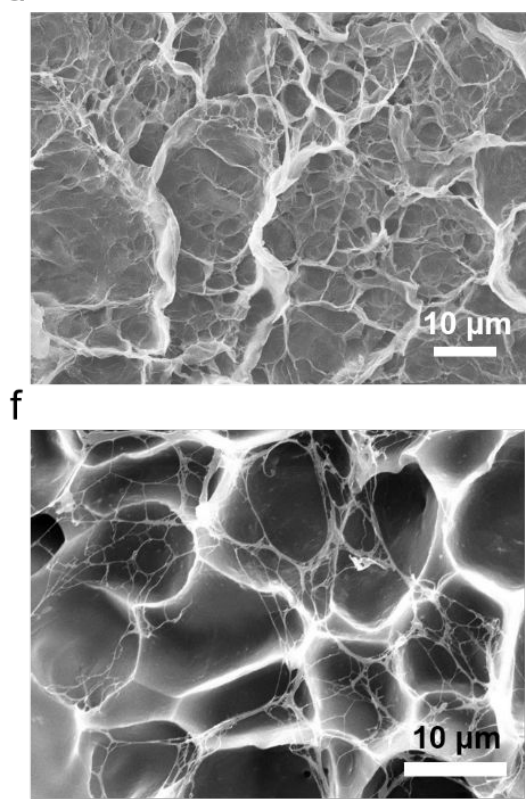

b

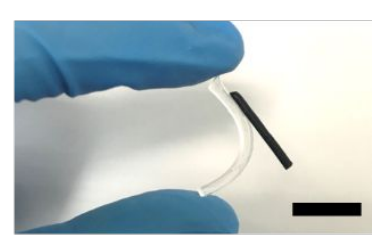

c

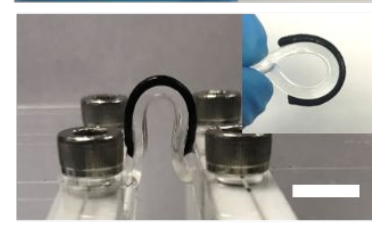

e

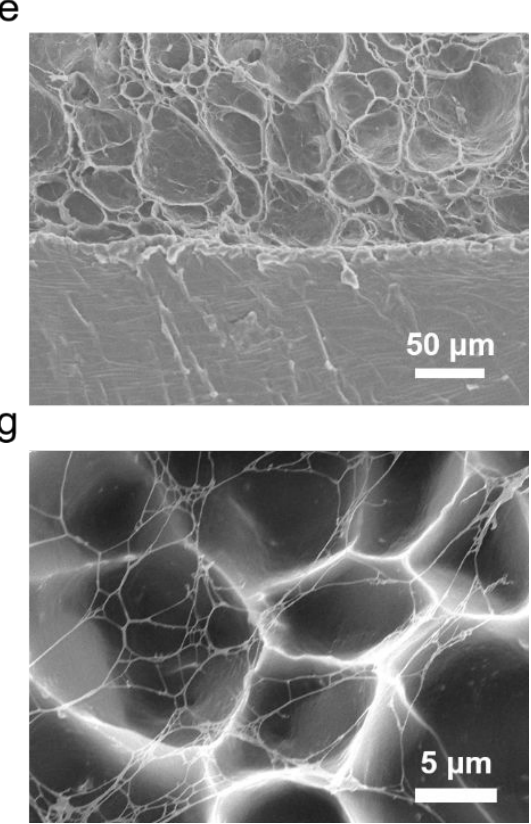

Figure S10. The robust interface bonding between hydrogel and PDMS. (a) Schematic diagram of the interface chemistry between PNIPAM hydrogel and PDMS. Two coupling agents TMSPAM and TEOVS are implanted into polymer chains of PNIPAM hydrogel and silicone rubber respectively, and then condense to form siloxane linkage upon contact. (b) PDMS-PNIPAM structure with no addition of silane coupling agents. A filament of NIPAM based DIW ink was printed (nozzle size of $1.8 \mathrm{~mm}$ ) on a PDMS strip $(2 \mathrm{~mm}$ in thickness) and cured. After bending, the PNIPAM filament detached from the interface. Scale bar is $10 \mathrm{~mm}$. (c) PDMS-PNIPAM structure with addition of silane coupling agents. A filament of NIPAM based DIW ink was printed (nozzle size of $1.8 \mathrm{~mm}$ ) on a PDMS strip $(2 \mathrm{~mm}$ in thickness) and cured. After bending to radius of $2 \mathrm{~mm}$, no detachment was observed. Scale bar is $10 \mathrm{~mm}$. (d) SEM image of the printed hydrogel from PNIPAM based ink. (e) SEM image of the interface of the PNIPAM based hydrogel (top) and the PDMS substrate (bottom). (f-g) SEM images of the hydrogel printed from NIPAM based ink with MWCNT addition. The MWCNT network is well dispersed and interconnected within the porous polymer matrix. 


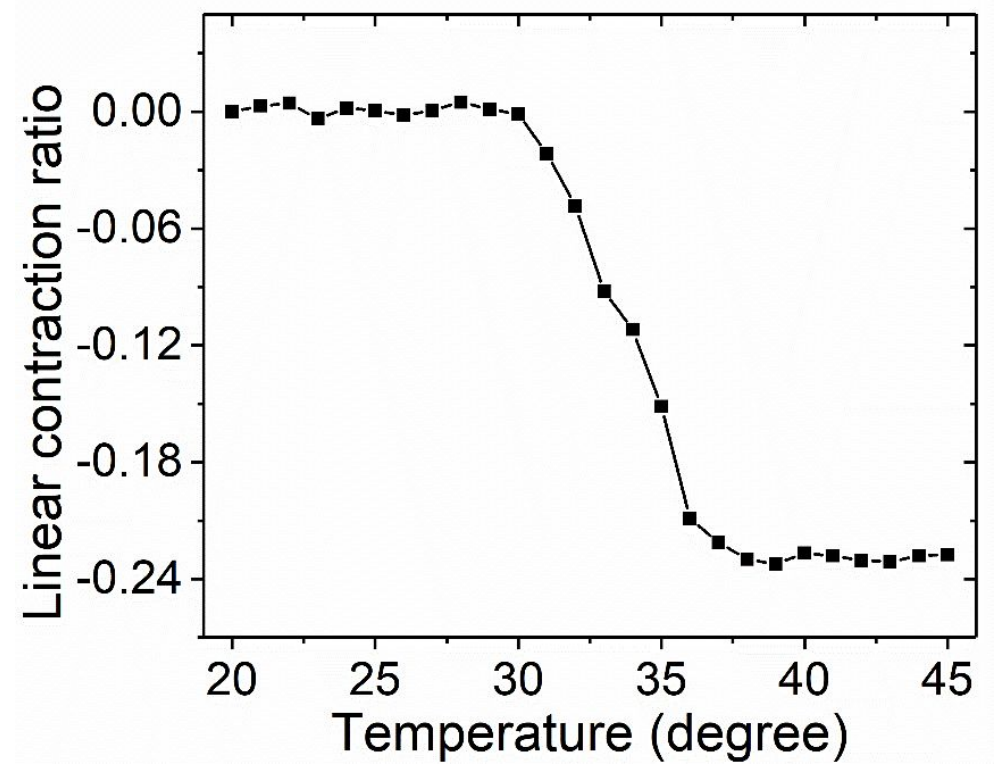

Figure S11. The linear contraction of the printed PNIPAM based hydrogel. To determine the linear contraction ratio, a filament of printed PNIPAM hydrogel $(8 \mathrm{~cm}$ in length) was immersed in DI water of $20^{\circ} \mathrm{C}$. The water temperature was then raised by one degree each time, till $45^{\circ} \mathrm{C}$. At each temperature point, the time was maintained for at least 30 mins, to allow the swelling equilibrium of the hydrogel. The length of the hydrogel was recorded along with the temperature variation. The hydrogel exhibited a transition temperature of $\sim 31{ }^{\circ} \mathrm{C}$, and a linear contraction ratio of $\sim 23 \%$. 

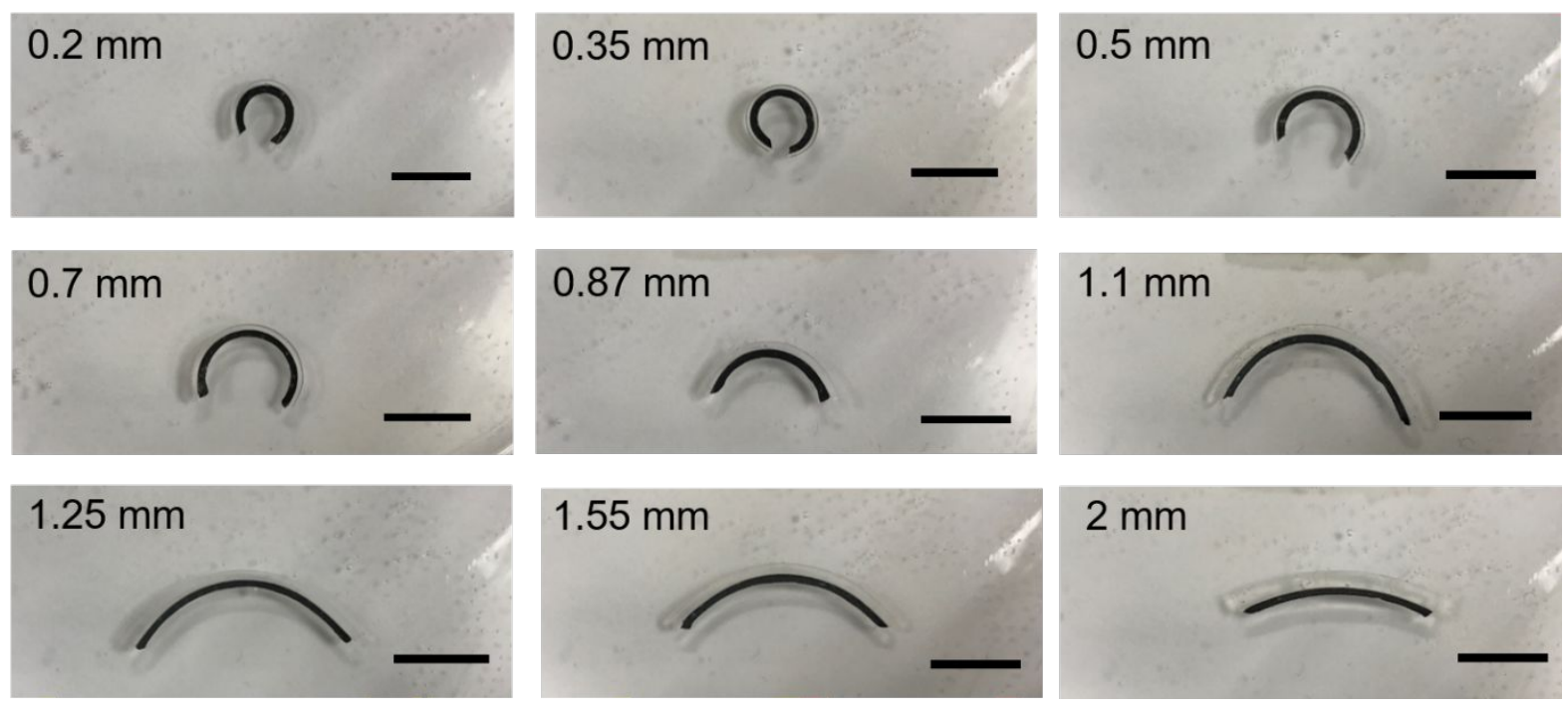

Figure S12. Bending of PDMS-PNIPAM bilayer structures. A series of PDMS-PNIPAM bilayer structures were prepared by printing (nozzle size of $0.84 \mathrm{~mm}$ ) photo-responsive PNIPAM hydrogel filaments on PDMS substrates (width of $1 \mathrm{~mm}$ ) with varying thickness ranging from 0.2 to $2 \mathrm{~mm}$. The PDMS-PNIPAM bilayer structures were soaked in water of $40{ }^{\circ} \mathrm{C}$ for at least 30 mins to reach swelling equilibrium, then the corresponding bending curvature was calculated through graphic processing. The results show that the bending curvature decreases as the PDMS thickness increases. Scale bars are all $10 \mathrm{~mm}$. 

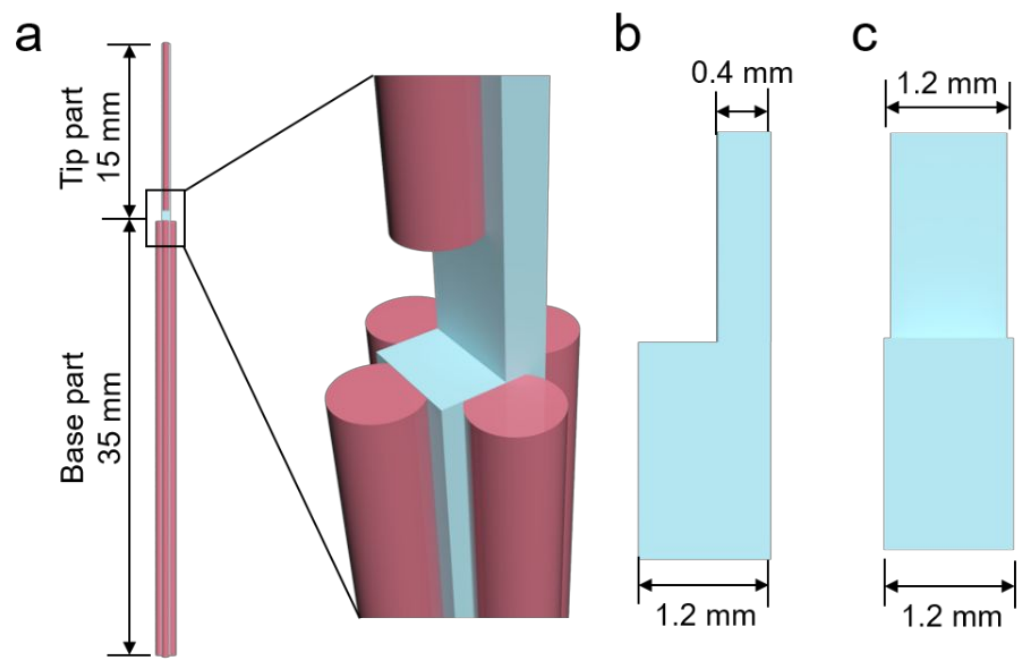

Figure S13. The geometrical design of the artificial tendril. (a) The overall architecture of the artificial tendril, including the tip part of $15 \mathrm{~mm}$ in length and the base part of $35 \mathrm{~mm}$ in length. (b) The side view of the PDMS layer. The thickness of the tip part and the base part is 0.4 and $1.2 \mathrm{~mm}$ respectively. (c) The front view of the PDMS layer. The width of the PDMS layer is $1.2 \mathrm{~mm}$. The photo-responsive PNIPAM hydrogel filaments are printed (nozzle size of $0.84 \mathrm{~mm}$ ) on one side of the tip part and 4 sides of the base part. The PDMS substrate was obtained from a 3D printed mold. 

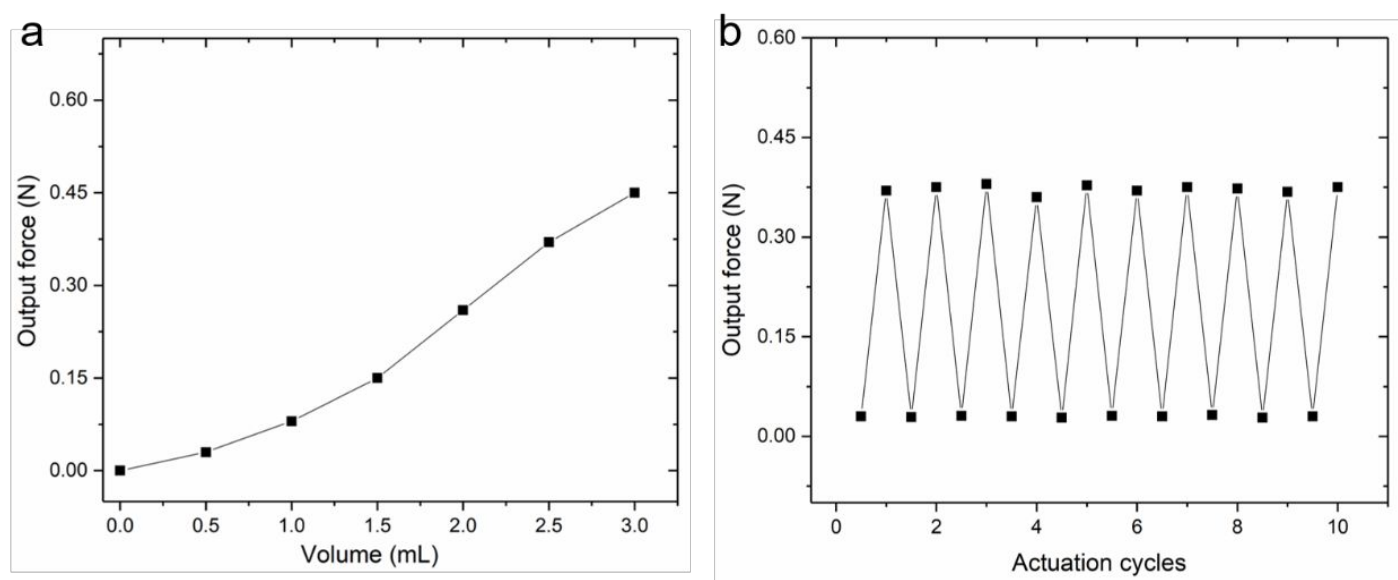

Figure S14. Output force at one end of the artificial tentacle in actuation. To characterize the output force of the artificial tentacle during actuation, we fixed the upper end of the tentacle to keep it hang vertically. A customized 1lb force gauge was brought into contact with the bottom part of the tentacle and fixed. We used a syringe pump (LEGATO270P, KDScientific) to control the injected air volume into one channel of the tentacle to bend to the force gauge direction. (a) The output force of the tentacle under increasing injected air volume. (b) The output force of the tentacle at air volume of 0.5 and $2.5 \mathrm{~mL}$ in 10 actuation cycles. 


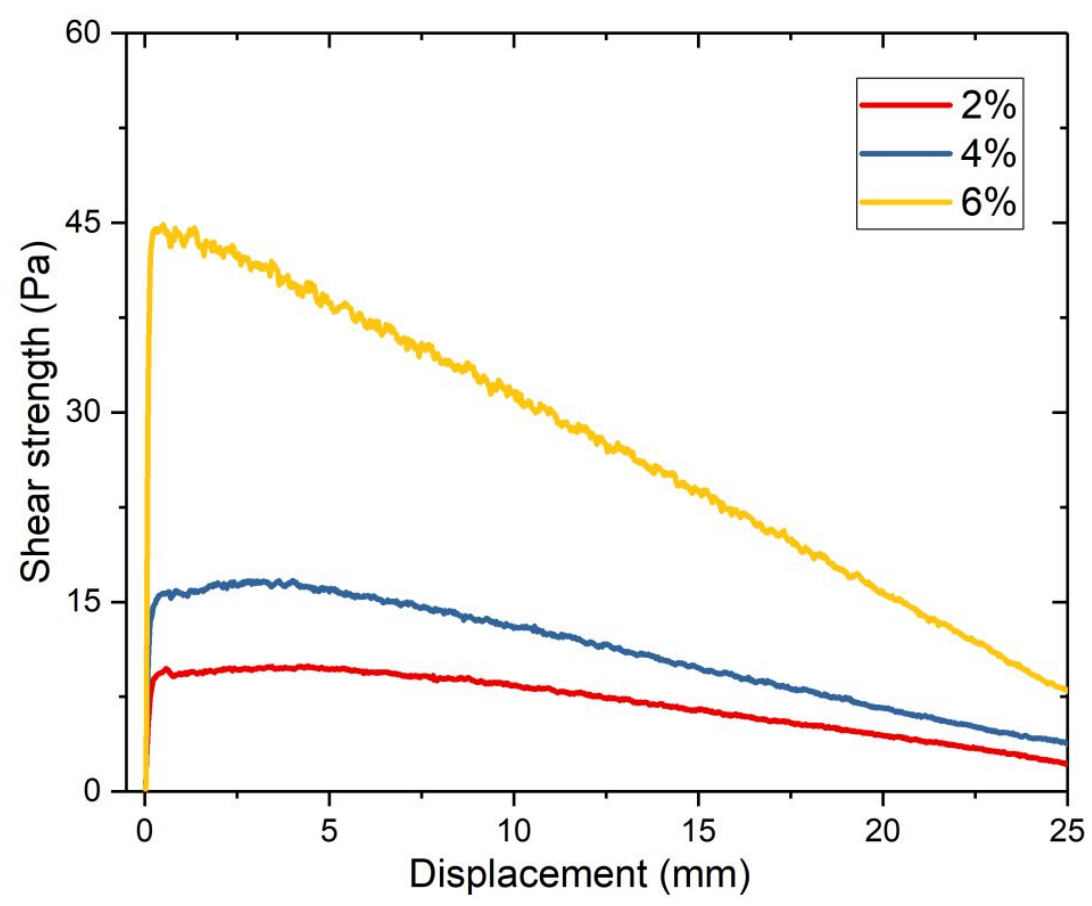

Figure S15. Lap shear test for the evaluation of the adhesion strength of the PNIPAM inks with alginate amount of $2,4,6 \mathrm{wt} \%$ to the PDMS substrate. The adhesion strength was tested according to the modified ASTM F2255-05 standard. Two glass slides $(25 \mathrm{~mm} \times 75 \mathrm{~mm})$ were used to hold each ink sample. The top portion of the glass slides $(25 \mathrm{~mm} \times 30 \mathrm{~mm})$ was coated with PDMS layer. The NIPAM based inks were added to one of the PDMS-coated region, and then the other slide was carefully put on above. After assembly, the two glass slides were placed into a customized tester for shear testing by tensile loading with a strain rate of $\mathbf{1 2 . 5}$ $\mathrm{mm} / \mathrm{min}$. The maximal detachment stress of the ink from the substrate was recorded as the adhesive strength. The test result exhibited that the adhesion strength of the NIPAM based ink increased with the increase of alginate amount, and the shear strength is 45 Pa for the 6 wt\% alginate ink for the printing of artificial tendril. 
a

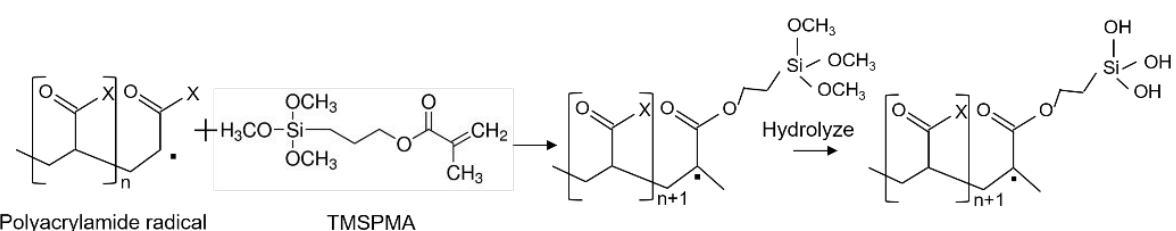

b

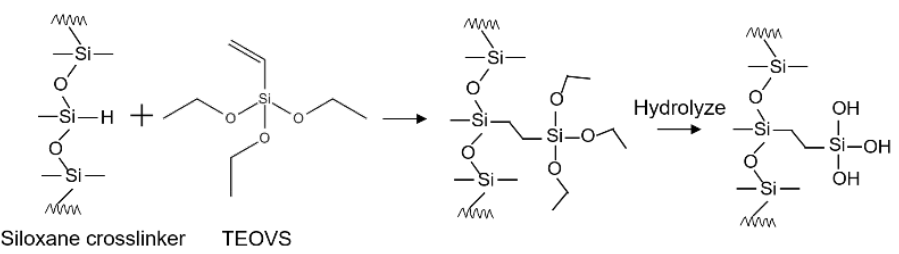

C

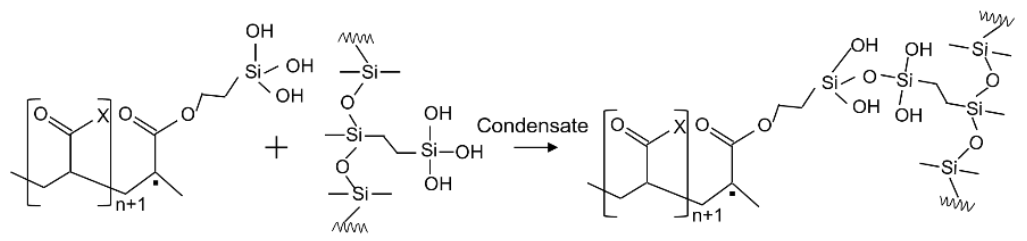

Figure S16. The chemical process of the silane coupling agents assisted interface bonding between PNIPAM hydrogel and PDMS. (a) The incorporation of TMSPMA into polyacrylamide network and hydrolysis. (b) The incorporation of TEOVS into PDMS network and hydrolysis. (c) The condensation of the two hydrolyzed silane coupling agents to form the siloxane bond.

\section{REFERENCES}

1. Timoshenko, S. Analysis of Bi-Metal Thermostats. J. Opt. Soc. Am. 1925, 11, 233-255. 